\title{
The Prevalence of ESBLs Producing Klebsiella pneumoniae Isolates in Some Major Hospitals, Iran
}

\author{
Sobhan Ghafourian ${ }^{1,2}$, Zamberi bin Sekawi ${ }^{1}$, Nourkhoda Sadeghifard ${ }^{2, *}$, Reza Mohebi ${ }^{2}$, \\ Vasantah Kumari Neela ${ }^{1}$, Abbas Maleki², Ali Hematian ${ }^{3}$, Mohammad Rahbar ${ }^{4}$, \\ Mohammad Raftari ${ }^{5}$ and Reza Ranjbar ${ }^{6}$ \\ ${ }^{I}$ Department of Medical Microbiology, and Parasitology, Faculty of Medicine and Health Science, University Putra Malaysia \\ ${ }^{2}$ Clinical Microbiology Research Center, Ilam University of Medical Sciences, Ilam, Iran \\ ${ }^{3}$ Department of Medical Microbiology, Ilam University of Medical Sciences, Ilam, Iran \\ ${ }^{4}$ Reference Laboratory of Iran \\ ${ }^{5}$ Faculty of Food Science and Technology, University Putra Malaysia, Malaysia \\ ${ }^{6}$ Molecular Biology Research Center, Baqiyatallah University of Medical Sciences, Tehran, Iran
}

\begin{abstract}
Aims of this study were to investigate on antibiotic resistance and molecular epidemiology of K.pneumoniae producing ESBLs isolates of respiratory tract infections in some major hospitals in Iran. K.pneumonaie were obtained of patients with RTI. K. pneumoniae producing ESBLs detected by screening, confirming and PCR methods. During the 12-month period, a total of one hundred and thirteen of K.pneumoniae were found from RTI in three cities in different region of Iran which Sixty seven strains $(59.2 \%)$ were ESBLs producer. In Ilam hospitals, seventeen strains (43.6\%), in Milad hospital, thirty-seven strains (74\%) and in Emam Reza hospital, thirteen strains (54.2\%) were ESBLs producer. The findings showed that among sixty-seven K.pneumoniae producing ESBLs, Sixty-three strains (94\%) were positive for blaSHV, eleven strains (16.4\%) contained blaTEM and sixteen strains (23.9\%) harbored blaCTX-M. Imipenem was found as an effectiveness antibiotic. In the current study, Majority of the ESBLs production had occurred in Milad hospital in Tehran (74\%). In conclusion, spreading ESBL-producing strains is a concern, as it causes limitations to the antimicrobial agents for optimal treatment of patients.
\end{abstract}

Keywords: ESBLs, Klebsiella pneumoniae, blaSHV, blaTEM, blaCTX-M.

\section{INTRODUCTION}

Extended-spectrum beta-lactamase (ESBL)-producing Klebsiella pneumoniae have spread rapidly worldwide and pose a serious threat in healthcare-associated infections [1]. ESBLs have spread threateningly in many regions of the world and now comprise over three hundred variants (http://www.lahey.org/studies).

ESBLs are plasmid-mediated enzymes that hydrolyze broad-spectrum beta-lactams and are strongly inhibited by clavulanate. ESBLs are transmitted by plasmids among bacteria. Furthermore, antibiotics such as trimethoprimsulfamethoxazole, aminoglycosides and fluoroquinolones are often co-transferred on a resistance plasmid, resulting in multiple drug resistance. Thus clinical treatment failure occurs frequently, especially when inappropriate antimicrobial therapy is used to treat infections caused by ESBL- producing organisms. Therefore, if infections with ESBL-producing organisms can be predicted by the clinical characteristics of patients, this may lead to a better selection of antibiotics and may improve the outcome of infections [2].

*Address correspondence to this author at the Clinical Microbiology Research Center, Ilam university of Medical, Sciences, Ilam, Iran;

Tel: 00989125146874; E-mail: sadeghifard@gmail.com
This study was done to investigate on antibiotic resistance and molecular epidemiology of $K$. pneumoniae producing ESBLs in patients with respiratory tract infections in some major hospitals in Iran.

\section{MATERIAL \& METHODS}

Sample collection: one hundred and thirteen clinical isolates of K.pneumoniae were identified during Mar. 2007 to Apr. 2008 in five hospitals in three Iranian cities (Ilam in west of Iran, Tabriz in west north of Iran, and Tehran in center and capital of Iran). K. pneumoniae isolates were obtained of sputum, tracheal aspirates, bronchial washing and bronchoalveolar lavage. Collection of multiple samples of the same patient was, however, avoided in the database.

\section{Screening Stage}

Kirby-Bauer disk diffusion test by using Mueller-Hinton agar diminished zones of inhibition around $3^{\text {rd }}$ generation beta-lactam disks were considered suggestive of ESBL production. According to NCCLS (2005) the following antibiotics were used to indicate ESBL production: cefpodoxime (30 $\mu \mathrm{g})$, cefotaxime $(30 \mu \mathrm{g})$, ceftazidime $(30 \mu \mathrm{g})$, cefteriaxone $(30 \mu \mathrm{g})$ and aztreonam $(30 \mu \mathrm{g})[3]$. 


\section{Clavulanic Acid Association Test}

For the combined disk method, disks containing cefpodoxime $(30 \mu \mathrm{g})$, ceftazidime $(30 \mu \mathrm{g})$ and cefotaxime $(30 \mu \mathrm{g})$ with and without clavulanic acid $(10 \mu \mathrm{g})$, were used. The resulting inhibition zones were compared. The test was considered positive when the difference of zone diameters between the beta-lactam disk and disk containing antibiotic associated with clavulanic acid was $>5 \mathrm{~mm}[4]$.

\section{Effect of non Beta-Lactame Antibiotics Against Klebsiella pneumoniae}

Amikacin (Ak) (30ug), cotrimoxazol (Co) (30ug) ciprofloxacin (Cf) (30ug), imipenem (I) (30ug) were used among K.pneumoniae producing ESBLs toward non beta-lactam antibiotics [5]. All antibiotic disks were obtained in HiMedia Company in India.

\section{Molecular Methods}

\section{DNA Extraction}

K.pneumoniae Producing ESBLs were cultured in LB broth at $37^{\circ} \mathrm{C}$ overnight, and then DNA was extracted by using the DNA extraction kit (fermenrtase).

\section{Polymerase Chain Reaction (PCR)}

The polymerase chain reaction (PCR) was carried out by following primers: blaTEM (Forward 5-GAGTATCAACA TTTCCGTGTC-3, Reverse primer 5-TAATCAGTGAGG CAC CTTCTC-3), blaSHV (5'-AAGATCCACTATCGCCCA GCAG-3, Reverse 5-AAGATCCA CTATCGCCCAGCAG-3) [6] and blaCTX-M, (forward 5-ACGCTGTTGTTAGGAA GTG-3, reverse 5-TTGAGGCTGGGTGAAGT-3) [7].

\section{RESULTS}

During the 12-month period, a total of one hundred and thirteen of K.pneumoniae isolates were obtained from respiratory tract infection in three cities in different part of Iran.
While total of sixty-seven strains (59.2\%) produced ESBLs, numbers of forty-six strains $(40.8 \%)$ were non ESBLs producer. Generally, amongst ESBLs producer K.pneumoniae, all the strains were resistant to aztreonam and cefpodoxime. Cefotaxime $(68.6 \%)$ allocated the lowest resistant among third generation of cephalosporin. Imipenem were found as an effectiveness antibiotic while resistance to cotrimoxazol (35.8\%) was more than the others (Table 1). In non ESBLs K.pneumoniae strains, no resistance occurred among aztreonam, while the highest resistance observed in cefotaxime and ceftazidime (50\%). In this study, all the non ESBLs K.pneomoniae were susceptible to the whole non-beta lactam antibiotics (Table 2). In Ilam city in the west of Iran, thirtynine K.pneumoniae were found that seventeen strains (43.6\%) were ESBLs producer and twenty-two strains (56.4\%) were negative for ESBLs production; in Tehran city in capital of Iran (Milad hospital), of fifty K.pneumoniae, thirty-seven strains $(74 \%)$ were positive for producing ESBLs and thirteen strains (26\%) were negative on behalf of producing ESBLs and in Tabriz city in the west north of Iran (Emam Reza hospital), twenty-four K. pneumoniae obtained which thirteen strains $(54.2 \%)$ were ESBLs producer and eleven strains $(45.8 \%)$ were negative.

Among K.pneumoniae producing ESBLs, 25.4\% ( $\mathrm{n}=17)$, $55.2 \%(\mathrm{n}=37)$ and $19.4 \%(\mathrm{n}=13)$ strains were isolated in Ilam, Milad and Emam Reza hospitals, respectively (Table 1).

Results revealed that among sixty-seven K.pneumoniae producing ESBLs, Sixty-three strains (94\%) were positive for blaSHV, eleven strains (16.4\%) contained blaTEM and sixteen strains (23.9\%) harbored blaCTX-M. blaCTX-M and blaSHV together were present in fourteen strains $(20.9 \%)$, nine strains (13.4\%) carried both blaSHV and blaTEM, four isolates $(6 \%)$ were positive for blaTEM and blaCTX-M and finaly, four isolates (6\%) carried all three blaSHV, blaTEM and blaCTX-M.

In Milad hospital, all K.pneumoniae producing ESBLs were positive for blaSHV, eight strains $(21.6 \%)$ contained

Table 1. Antibiotic Panel of K.pneumoniae Producing ESBLs Strains

\begin{tabular}{|l|l|l|l|l|}
\hline \multirow{2}{*}{ Antibiotics } & $\begin{array}{l}\text { ESBLs Positive } \\
\text { K.pneumoniae } \\
\text { In Ilam Hospital } \\
\text { N=17 }\end{array}$ & $\begin{array}{l}\text { ESBLs Positive K.pneumoniae } \\
\text { In Emam Reza Hospital } \\
\text { N=13 }\end{array}$ & $\begin{array}{l}\text { ESBLs Positive K.pneumoniae } \\
\text { In Milad Hospital } \\
\text { N=37 }\end{array}$ & $\begin{array}{l}\text { ESBLs Positive K.pneumoniae } \\
\text { Total } \\
\text { N=67 }\end{array}$ \\
\cline { 2 - 5 } & Resistance \% Sensitivity\% & Resistance \% Sensitivity\% & Resistance \% Sensitivity\% & Resistance \% Sensitivity\% \\
\hline \hline Ca & $14(82 \%) 3(18 \%)$ & $13(100 \%) 0$ & $35(94.6 \%) 2(5.4 \%)$ & $62(92.5 \%) 5(7.5 \%)$ \\
\hline Ce & $6(35.2 \%) 11(64.8 \%)$ & $12(92.3 \%) 1(7.7 \%)$ & $28(75.7 \%) 9(24.3 \%)$ & $46(68.6 \%) 21(31.3 \%)$ \\
\hline Ci & $17(100 \%) 0$ & $13(100 \%) 0$ & $32(86.5 \%) 5(13.5 \%)$ & $62(92.5 \%) 5(7.5 \%)$ \\
\hline Cep & $17(100 \%) 0$ & $13(100 \%) 0$ & $37(100 \%) 0$ & $67(100 \%) 0$ \\
\hline Ao & $17(100 \%) 0$ & $13(100 \%) 0$ & $37(100 \%) 0$ & $67(100 \%) 0$ \\
\hline Ak & $3(18 \%) 14(82 \%)$ & $3(23.1 \%) 10(76.9 \%)$ & $13(35.1 \%) 24(64.9 \%)$ & $19(28.3 \%) 48(71.6 \%)$ \\
\hline Cf & $2(12 \%) 15(88 \%)$ & $1(7.7 \%) 12(92.3 \%)$ & $8(21.6 \%) 29(78.4 \%)$ & $11(16.4 \%) 56(83.6 \%)$ \\
\hline Co & $7(41 \%) 10(59 \%)$ & $3(23.1 \%) 10(76.9 \%)$ & $14(37.9 \%) 23(62.1 \%)$ & $24(35.8 \%) 43(64.1 \%)$ \\
\hline I & $017(100 \%)$ & $013(100 \%)$ & $037(100 \%)$ & $067(100 \%)$ \\
\hline
\end{tabular}


Table 2. Antibiotic Panel of K.pneumoniae non-ESBLs Strains

\begin{tabular}{|l|l|l|l|l|}
\hline \multirow{2}{*}{ Antibiotics } & $\begin{array}{l}\text { ESBLs Negative K.pneumoniae } \\
\text { In Ilam Hospital } \\
\text { N=22 }\end{array}$ & $\begin{array}{l}\text { ESBLs Negative K.pneumoniae } \\
\text { In Emam Reza Hospital } \\
\text { N=11 }\end{array}$ & $\begin{array}{l}\text { ESBLs Negativ K.pneumoniae } \\
\text { In Milad Hospital } \\
\text { N=13 }\end{array}$ & $\begin{array}{l}\text { ESBLs Negative K.pneumoniae } \\
\text { Total } \\
\text { N=46 }\end{array}$ \\
\cline { 2 - 5 } & Resistance \% Sensitivity\% & Resistance \% Sensitivity\% & Resistance \% Sensitivity\% & Resistance \% Sensitivity\% \\
\hline Ca & $11(50 \%) 11(50 \%)$ & $6(54.5 \%) 5(45.6 \%)$ & $6(46.2 \%) 7(53.8 \%)$ & $23(50 \%) 23(50 \%)$ \\
\hline Ce & $9(40.9 \%) 13(59.1 \%)$ & $4(36.3 \%) 7(63.7 \%)$ & $10(76.9 \%) 3(23.1 \%)$ & $23(50 \%) 23(50 \%)$ \\
\hline Ci & $12(54.5 \%) 10(45.6 \%)$ & $5(45.5 \%) 6(54.5 \%)$ & $5(38.5 \%) 8(61.5 \%)$ & $22(47.9 \%) 24(52.1 \%)$ \\
\hline Cep & $1(4.5 \%) 21(94.5 \%)$ & $1(9 \%) 10(90.9 \%)$ & $013(100 \%)$ & $2(4.34 \%) 44(95.6 \%)$ \\
\hline Ao & $022(100 \%)$ & $011(100 \%)$ & $013(100 \%)$ & $046(100 \%)$ \\
\hline Ak & $022(100 \%)$ & $011(100 \%)$ & $013(100 \%)$ & $046(100 \%)$ \\
\hline Cf & $022(100 \%)$ & $011(100 \%)$ & $013(100 \%)$ & $046(100 \%)$ \\
\hline Co & $022(100 \%)$ & $011(100 \%)$ & $013(100 \%)$ & $046(100 \%)$ \\
\hline I & $022(100 \%)$ & $011(100 \%)$ & $046(100 \%)$ \\
\hline
\end{tabular}

blaTEM and ten isolates (27\%) carried blaCTX-M. blaSHV and blaCTX-M presented in ten $(27 \%)$ of strains. Eight strains $(21.6 \%)$ carried both blaSHV and blaTEM. Four isolates were positive for both blaTEM and blaCTX-M and all three genes were found in four isolates $(10.8 \%)$.

In Ilam hospital, among seventeen K.pneumoniae producing ESBLs, blaSHV was found in thirteen strains (76.5\%), two strains $(11.8 \%)$ were positive for blaTEM, and blaCTX-M presented in five strains $(29.4 \%)$. Three strains (17.7\%) carried both blaSHV and blaCTX-M.

In Emam Reza hospital, all strains were positive for blaSHV, while one strain carried blaTEM and one isolate was positive for blaCTX-M and one for both blaSHV and blaCTX-M, as well.

\section{DISCUSSION}

The high rate of ESBLs among hospitalized patients is a global problem. It is generally thought that patients infected by an ESBL-producing organism are at an increased risk of treatment failure with an expanded-spectrum beta-lactam. The prevalence of ESBL producing isolates of $K$. pneumoniae varies in different countries [8].

Countries with a high rate of prevalence include Turkey (60\%), Latin America (45.4\%), Western Pacific (24.6\%), and Europe (22.6\%) [9].

In this study, ESBLs production was variable from $43.6 \%$ in Ilam hospital to $74 \%$ in Milad hospital that showed different frequency of ESBLs production in different region in Iran. Our study showed significantly high ESBLs production.

The prevalence of respiratory isolates of $K$. pneumoniae with ESBL phenotype has been reported from less than $1 \%$ in Japan [10] to $83.3 \%$ in China [11].

We showed that the percentage of respiratory isolates with $K$. pneumoniae was high. Milad hospital contributed to more of the K.pneumoniae isolates. The lowest K.pneumoniae producing ESBLs had observed in Ilam hospital. Nonbeta-lactam antibiotic resistance in Milad hospital was more than the others. Imipenem was found as an effectiveness antibiotic.

In this study, susceptibility testing of K.pneumonie strains producing ESBLs showed that the highest resistance rate among $3^{\text {rd }}$ generation of cephalosporins and aztreonam were cefpodoxime and aztreoname in all hospitals, cefteriaxone in Emam Reza and Ilam hospitals and ceftazidime in Emam Reza hospital. The highest non-beta lactam antibiotic resistance occurred in cotrimoxazol in Ilam hospital. Imipenem was found as effectiveness antibiotic among ESBLs producing K.pneumoniae strains.

Majority of the ESBLs production recurred in Milad hospital $(74 \%)$. The highest resistance toward non-beta-lactam antibiotic was observed in cotrimoxazol $(41 \%)$ in Ilam hospital. The best antibiotics were Imipenem (100\%) in all hospitals and following ciprofloxacine $(92.3 \%)$ in Emam Reza Hospital. In non-ESBLs, K.pneumoniae Resistance toward cefotaxime $(76.9 \%)$ was more than the other antibiotics. The most genes responsible for ESBLs production were found in blaSHV. In Ilam Hospital, frequency of blaCTX-M was more than blaTEM.

In Milad Hospital, Frequency of blaCTX-M was more than blaTEM.

Our finding in Emam Reza hospital had showed frequency of blaTEM and blaCTX-M were as an equal.

The prevalence of blaSHV,blaTEM and blaCTX-M genes in this study was $94 \%, 16 \%$ and $23.9 \%$, respectively.

In Iran, Feizabadi et al., in 2009 showed that $69.7 \%$ of $K$. pneumoniae isolates in Tehran were ESBL producers and the prevalence of blaTEM, blaSHV, blaCTX-M-I and blaCTXM-III among these isolates were 54\%, 67.4\%, $46.51 \%$ and 
$29 \%$, respectively (12). Our results revealed high prevalence of blaSHV and low frequency of blaTEM and blaCTX-M. Significantly, blaSHV was more responsible for ESBLs production [12].

In the study a tertiary care hospital in Tehran $77 \%$ of K.pneumoniae were ESBLs producer [13] while in our study in Ilam and Emam Reza hospitals frequency of ESBLs production were lower while the results of Milad hospital (74\%) was near to the study of mehregan et al., [13]. In a survey by bazzaz et al., in 2007 in general hospital in Iran $59.2 \%$ of isolates were positive for ESBLs production and all isolates were susceptible to imipenem [14], our finding showed all strains were susceptible to imipenem. Our findings in Milad hospital revealed higher ESBLs production as comparison to bazzaz et al., [14].

Shahcheragi et al., [6] showed that ESBLs production observed in 33\% of isolates while in our study the lowest ESBLs production was presented in Ilam hospital (43.6\%). All isolates in survey of shahcheraghi et al., [6] were susceptible to imipenem and ciprofloxacine resistance was observed in $32 \%$ of K.pneumoniae. our finding revealed all strain were susceptible to imipenem but the highest rate of resistant to ciprofloxacine was observed in Milad hospital and that was $21.6 \%$. In the study of shahcheraghi et al., $69.6 \%$ of strains carried blaSHV and $32.1 \%$ of K.pneumonie producing ESBLs harbored blaTEM [6] while our results showed all strains in Emam Reza and Milad hospitals carried blaSHV and this was $76.5 \%$ in Ilam hospital (94\%). Frequency of blaTEM was lower (16.4\%) than survey of shahcheraghi et al., in this study we found different ESBLs production in different regions of Iran that one of the reason may related to population in different city and also Using of antibiotic in Iran is uncontrollable, use of antibiotics and injectable formulations was high in the Islamic Republic of Iran. The high number of prescriptions for antibiotics $(58 \%$ on average) may be because in the majority of the provinces, the data collected only covered a period of 1 month. And different prescriptions may have result of different resistance to antibiotics [15]. In conclusion, spreading ESBL-producing strains is a concern, as it causes limitations to the antimicrobial agents for optimal treatment of patients. The most reliable and effective antimicrobial treatment for infections caused by this organism is imipenem [16] and also had shown in this study. BlaSHV was found as a predominant gene responsible for ESBLs production and future study need to determine type of bla genes responsible for ESBLs producing strains in Iran and more study in different part of Iran.

\section{ACKNOWLEDGEMENT}

University Putra Malaysia and Ilam University of Medical Sciences provided partial support for the laboratory studies and interpretation.

\section{ABBREVIATIONS}

$\begin{array}{ll}\mathrm{Ak} & =\text { Amikacin } \\ \mathrm{Ao} & =\text { Aztreonam } \\ \mathrm{Ca} & =\text { Ceftazidim }\end{array}$

$\begin{array}{ll}\mathrm{Cac} & =\text { Ceftazidime/clavulanic } \\ \mathrm{Ce} & =\text { Cefotaxime } \\ \mathrm{Cec} & =\text { Cefotaxime /clavulanic } \\ \mathrm{Cep} & =\text { Cefpodoxim } \\ \mathrm{Cepc} & =\text { Cefpodoxim /clavulanic acid } \\ \mathrm{Cf} & =\text { Ciprofloxacin } \\ \mathrm{Ci} & =\text { Cefteriaxon } \\ \mathrm{Co} & =\text { Cotrimoxazol } \\ \mathrm{ESBL} & =\text { Extended spectrum beta-lactamases } \\ \mathrm{I} & =\text { Imipenem } \\ \text { K. pneumoniae } & =\text { Klebsiella pneumoniae } \\ \mathrm{RTI} & =\text { Respiratory tract infection }\end{array}$

\section{REFERENCES}

[1] Kiratisin P, Apisarnthanarak A, Laesripa C, Saifon P. Molecular characterization and epidemiology of extended-spectrum-betalactamase-producing Escherichia coli and Klebsiella pneumoniae isolates causing health care-associated infection in Thailand, where the CTX-M family is endemic. Antimicrob Agents Chemother 2008; 52:2818-24.

[2] Martinez-Martinez L, Pascual A, Jacoby GA. Quinolone resistance from a transferable plasmid. Lancet 1998: 351: 797-9.

[3] NATIONAL COMMITTEE FOR CLINICAL LABORATORY STANDARDS - NCCLS. M100-S13. Performance Standards for Antimicrobiak Susceptibility Testing; Thirteenth Informational Supplement. Wayne, Pennsylvania, USA: NCCLS documents, 2005.

[4] Jarlier V, Nicolas MH, Fournier G, Philippon A. Extended broadspectrum Beta-lactamases confering transferable resistance to newer Beta-lactam agents in Enterobacteriaceae hospital prevalence and susceptibility patterns. Rev Infect Dis Chigaco, 1998; v.10, p.867-878.

[5] Paterson DL, Mulazimoglu L, Casellas J M, et al. Epidemiology of ciprofloxacin resistance and its relationship to extended-spectrum beta-lactamase production in Kelbsiella pneumoniae isolates causing bacteremia. Clin Infect Dis 2000; 30: 473-8.

[6] Shahcheraghi F, Moezi H, Feizabadi M. Distribution of TEM and SHV Beta-lactamase genes among klebsiella pneumoniae strains isolated from patients in Tehran. Med Sci Moint 2007; 13:BR247-250.

[7] Mansouri M, Ramazanzadeh R. Spearead of extended spectrum beta-lactamases producing E.coli clinical isolates in sanandaj hospital. J Biol Sci 2009; 9; 362-36.

[8] Shah AA, Hasan F, Ahmed S, Hameed A. Characteristics, epidemiology and clinical importance of emerging strains of Gramnegative bacilli producing extended-spectrum beta-lactamases. Res Microbiol 2004; 155: 409-21.

[9] Gonlugur U, Bakici MZ, Akkurt I, Efeoglu T. Antibiotic susceptibility patterns among respiratory isolates of Gramnegative bacilli in a Turkish university hospital. BMC Microbiol 2004; 4: 32.

[10] Yum JH, Kim S, Lee H, et al. Emergence and wide dissemination of CTX-M-type ESBLs, and CMY-2- and DHA-1-type AmpC beta-lactamases in Korean respiratory isolates of Klebsiella pneumoniae. J Korean Med Sci 2005; 20: 961-5.

[11] Shi J, Li Y, Li C, Cai X, Li H, Peng S. Drug resistance and genotyping of Klebsiella pneumoniae in lower respiratory tract infection. Zhonghua Jie He He Hu Xi Za Zhi 2002; 25: 607-9.

[12] Feizabadi MM, Delfani S, Raji N, et al. Distribution of bla(TEM), bla(SHV), bla(CTX-M) genes among clinical isolates of Klebsiella pneumoniae at Labbafinejad hospital, Tehran, Iran. Microb Drug Resist 2010; 16:49-53.

[13] Mehrgan H, Rahbar M, Arab-Halvaii Z. High prevalence of extended-spectrum beta-lactamase-producing Klebsiella pneumoniae 
in a tertiary care hospital in Tehran, Iran. J Infect Dev Ctries 2010; 29; 4: 132-8.

[14] Bazzaz BS, Naderinasab M, Mohamadpoor AH, Farshadzadeh Z, Ahmadi S, Yousefi F. The prevalence of extended-spectrum betalactamase-producing Escherichia coli and Klebsiella pneumoniae among clinical isolates from a general hospital in Iran. Acta Microbiol Immunol Hung 2009; 56:89-99.
[15] Cheraghali AM, Nikfar S, Behmanesh Y. Evaluation of availability, accessibility and prescribing pattern of medicines in the Islamic Republic of Iran. East Mediterr Health J 2004; 10(3): 406-5.

[16] Essack SY. Treatment options for extended-spectrum betalactamase- producers. FEMS Microbiol Lett 2000; 190:181-4.

Received: June 02, 2011

Revised: June 20, 2011

Accepted: June 24, 2011

(c) Ghafourian et al.; Licensee Bentham Open.

This is an open access article licensed under the terms of the Creative Commons Attribution Non-Commercial License (http://creativecommons.org/licenses/ by-nc/3.0/) which permits unrestricted, non-commercial use, distribution and reproduction in any medium, provided the work is properly cited. 